Prolegómeno al concepto de Corrupción a Gran Escala: disertaciones desde la base social hacia su tipificación

Prolegomena to the concept of Large-scale Corruption: Dissertation from the social base towards its typification

Autores: Alex Garcés Medrano, Tirson Mauricio Duarte Molina y Juan David Mambuscay Burbano

DOI: https://doi.org/10.25058/1794600X.1953

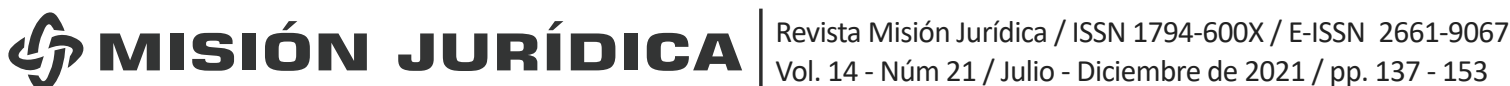




\title{
Prolegómeno al concepto de Corrupción a Gran Escala: disertaciones desde la base social hacia su tipificación*
}

\author{
Prolegomena to the concept of Large-scale Corruption: \\ Dissertation from the social base towards its typification \\ Prolegômeno ao conceito de Corrupção em Grande Escala: \\ dissertações da base social à sua tipificação
}

\author{
Alex Garcés Medrano ${ }^{a}$ \\ garcesmedrano@gmail.com \\ Tirson Mauricio Duarte Molina ${ }^{b}$ \\ tirsonmduarte@gmail.com \\ Juan David Mambuscay Burbano ${ }^{c}$ \\ mambuscay.jd@gmail.com
}

Fecha de recepción: 15 de enero de 2021

Fecha de revisión: 30 de marzo de 2021

Fecha de aceptación: 15 de abril de 2021

\section{DOI: https://doi.org/10.25058/1794600X.1953}

\section{RESUMEN}

Conceptuar el término de corrupción a nivel genérico ha suscitado diversas manifestaciones por parte de la doctrina, por medio de la cual se ha identificado el riesgo que surge a partir de esta, máxime cuando se traslada a gran escala, comprendiendo ello una afectación a las instituciones estatales así como también a su legitimidad. Situación que se ve reflejada en la configuración del acto corrupto en sí mismo, y en la incidencia que la consumación de este tiene a nivel público, al igual que en la esfera de lo privado, impacto que puede verse reflejado a través de los obstáculos sistematizados que atraviesan los Estados en la materialización de la función pública.

\footnotetext{
* Este artículo de investigación es producto del proyecto "La legitimidad institucional en Colombia: análisis al involucramiento del poder judicial en fenómenos de corrupción a gran escala" del Grupo de Investigación en Derecho y Ciencias Políticas de la Fundación Universidad Católica Lumen Gentium.

a. Alex Garcés Medrano. Abogado, politólogo, magister en filosofía del derecho contemporáneo, doctorando en ciencias sociales y jurídicas. Docente universitario. Correo: garcesmedrano@gmail.com ORCID: 0002-8537-9448.

b. Tirson Mauricio Duarte Molina. Abogado de la Universidad de San Buenaventura - Cali. Estudiante Especialización en Derecho Administrativo de la Universidad Libre de Colombia - Seccional Cali. Becario Maestría en Derecho de la Universidad Icesi. Miembro de la Red Latinoamericana de Metodología de las Ciencias Sociales - RedMet. Investigador en Diacronía: Centro de Estudios Sociales y Humanísticos. Correo: tirsonmduarte@gmail.com. ORCID: 0002-6240-2609.

c. Juan David Mambuscay Burbano. Profesional en Gobierno y Relaciones Internacionales de la Universidad de San Buenaventura - Cali. Miembro de la Asociación Colombiana de Ciencia Política - ACCPOL. Cursó "Gestión de Proyectos Sociales para ONG y Organizaciones de la Sociedad Civil (OSC) del Banco Interamericano de Desarrollo. Investigador en Diacronía: Centro de Estudios Sociales y Humanísticos. Correo: mambuscay.jd@gmail.com. ORCID: 0003-1866-7244
} 
De este modo, y a partir de su categorización, surge la corrupción a gran escala como una variante del fenómeno de acto corrupto per se, fungiendo así como una amenaza al aparato estatal, esto es, al poder público, toda vez que el desarrollo de esta comprende dentro de sí la posibilidad de la comisión de una mayor variedad de conductas punibles por parte de sus actores. Ahora bien, es importante resaltar que la identificación, que se ha realizado previamente de tal figura, ha sido insuficiente, no en vano la tipificación de esta conducta a nivel legislativo, a partir de elementos únicamente técnicoadministrativos, representa cierta ignorancia en lo que a las prácticas de carácter deliberativo, por parte de los sujetos al interior de los regímenes democráticos, se refiere.

Por tal razón, la presente investigación tiene por objetivo la recopilación de los elementos teóricos que pueden encontrarse en torno a la Corrupción a Gran Escala, fundamentándose para ello de forma enfática en el estado de la cuestión, para luego, realizar un análisis exhaustivo de los factores que resultan fundamentales al interior de la teoría de la acción comunicativa, comprendida como piedra angular de la deliberación en sociedad, para, finalmente, instaurar un planteamiento de oportunidades que la Corrupción a Gran Escala brinda de cara al fortalecimiento de la democracia.

\section{PALABRAS CLAVES}

Corrupción a Gran Escala; legitimidad; democracia; normas; acción comunicativa; derechos; legalidad.

\section{ABSTRACT}

The conceptualization of the notion of corruption at a generic level has given rise to various doctrine manifestations, by means of which the risk that arises from it has been identified, especially when it is transferred on a large scale, including an impact on state institutions as well as their legitimacy. This situation is reflected in the configuration of the corrupt act itself, and in the impact that its consummation has on the public level, as well as in the private sphere, an impact that can be reflected through the systematized obstacles that the States go through in the materialization of the public function.
Thus, and from its categorization, Largescale Corruption emerges as a variant of the phenomenon of corrupt act per se, therefore serving as a threat to the state apparatus, that is, to public power, since the development of it includes the possibility of the carrying out of a greater variety of punishable conducts by its actors. However, it is important to emphasize that the identification that has been previously made of such a figure has been insufficient, not in vain the typification of this conduct at the legislative level, based only on technical-administrative elements, represents a certain ignorance regarding to the deliberative practices of the subjects within the democratic regimes.

For this reason, the objective of this research is to compile the theoretical elements that can be found in relation to Large Scale Corruption, basing itself emphatically on the state of the question, to subsequently carry out an exhaustive analysis of the factors that are fundamental within the Theory of Communicative Action, understood as the cornerstone of deliberation in society, to finally establish an approach to the opportunities that Large-scale Corruption offers for strengthening democracy.

\section{KEY WORDS}

Large Scale Corruption; Legitimacy; Democracy; Norms; Communicative Action; Rights; Legality.

\section{RESUMO}

A conceituação do termo corrupção em um nível genérico tem levado a diversas manifestações por parte da doutrina, por meio das quais foi identificado o risco que dela advém, especialmente quando é transferida em grande escala, inclusive com impacto nas instituições do estado como bem como sua legitimidade. Situação que se reflete na configuração do próprio ato corrupto, e no impacto que sua consumação tem na esfera pública, assim como na esfera privada, impacto que pode se refletir nos entraves sistematizados pelos quais passam os Estados no a materialização da função pública.

Desse modo, e a partir de sua categorização, surge a corrupção em larga escala como uma variante do fenômeno do ato corrupto per se, agindo assim como uma ameaça ao aparelho de Estado, ou seja, ao poder público, desde o desenvolvimento deste. inclui em si a 
possibilidade de cometimento de uma maior variedade de comportamentos puníveis por seus atores. Ora, é importante destacar que a identificação, que se fez anteriormente de tal figura, tem sido insuficiente, não em vão a tipificação desse comportamento a nível legislativo, com base apenas em elementos técnicoadministrativos, representa um certo ignorância no que se refere às práticas deliberativas de indivíduos em regimes democráticos.

Por esse motivo, a presente pesquisa tem como objetivo compilar os elementos teóricos que podem ser encontrados em torno da Corrupção em Grande Escala, enfaticamente a partir do estado da questão, e realizar uma análise exaustiva dos fatores fundamentais dentro da teoria da ação comunicativa, entendida como uma pedra angular da deliberação na sociedade, para finalmente estabelecer uma abordagem às oportunidades que a Corrupção em Grande Escala oferece para o fortalecimento da democracia.

\section{PALAVRAS-CHAVES}

Corrupção em grande escala; legitimidade; democracia; as regras; ação comunicativa; Direitos; legalidade.

\section{INTRODUCCIÓN}

Analizar los espacios de deliberación habilitados para la ciudadanía, como momentos fundamentales en la concertación y el establecimiento de postura ante asuntos considerados de importancia común y, más allá, como ejercicio fundamental en el proceso de autodeterminación ciudadana frente a la toma de decisiones racionales y justas (Michelini, 2015) trae consigo el abordaje, en sí, de la noción de Estado Social de Derecho, visto como el modelo constitucional propio de los regímenes democráticos occidentales, en donde se dota a los ciudadanos de un compendio de derechos entorno a la igualdad, la participación y la equidad (Bueno y Manzur, 2012).

Respaldados en este andamiaje institucional, las distintas formas (cuales sea que se tomen) de los citados momentos, generan consigo, a su vez, la convergencia, así como la divergencia, de distintas vivencias, toda vez que éstas últimas se desarrollan en función de valores, prácticas, costumbres y reglas comprendidas desde la geografía vital (Guitart, 2011) y que, tienen paso mediante las ideas, además de la interacción entre los agrupados.

Con base a lo anterior, no funge como idea poco elocuente el afirmar que, tras la concepción de leyes y convenciones que regulen los problemas y conflictos en la comunidad, el aspecto plural de la condición democrática (en referencia a los ejercicios que en ella se gesten) hace las veces de insumo con el que estas se nutren, delimitando la deliberación y/o participación de los sujetos tal cual dinámica esencial, no solo en la identificación de los aspectos a resolver, sino también en qué se entiende por ellos; desde aquí, el diálogo acoge su instante legitimador, entendida la conformación de aquello que regula como bien último por alcanzar.

De cara a ésta última idea, el análisis que suscita la corrupción, comprendida entre los fenómenos que develan enormes retos para los Estados y su capacidad institucional, así vista igualmente como "un comportamiento que abusa o se desvía de los deberes formales de un rol público/poder público/cargo público, para obtener un beneficio o ganancia privada (personal o para un tercero)" (Pont y Arango, 2017, p. 35) trae consigo también sus variantes, entre las que, como objeto de estudio, se identifica la Corrupción a Gran Escala (CAGE, de ahora en adelante). La complejidad de esta tipología, descansa no solo en el poco desarrollo académico (y por menos, judicial) que ha tenido, sino de la misma forma, en la ambigüedad que la categoría general -Corrupción- posee; ya Gómez-Díaz (2003) caracteriza la corrupción no solamente desde el actuar público, sino también desde la omisión, situación desde la cual se pueden obtener ventajas de tipo pecuniarios, información privilegiada, etc., en tanto Pont y Arango (2017) reflejan las distintas denominaciones que la literatura académica se ha encargado de construir; cada una de ellas se establece en función de varios elementos entre los que se encuentran: el impacto y los actores involucrados.

En este contexto, qué entender por CAGE, a merced de su incipiente desarrollo, situación contraria hallada en los resultados que de ella persisten en la actualidad sobre el grueso social, abre las puertas a un momento de deliberación en el que se fortalezca la democracia, producto de la deliberación realizada por los individuos vinculados y la exposición de sus ideas al 
respecto, todo ello, previo a las instancias técniconormativas que tipifiquen este fenómeno como delito. Así, por un lado, se genera el insumo de legitimación ante las decisiones tomadas, mientras que, por el otro, la legitimidad es agrupada en la postura misma que el cuerpo institucional adquiere de cara al problema que, según ha de ser mostrado, involucra negativamente en demasía el bienestar de la sociedad una vez se arraiga al sistema político.

Visto así, la CAGE puede ser interpretada desde una dualidad amenaza-oportunidad para la democracia. Luego pues, comprendiendo que la primera arista está en claro cómo tiene lugar, surge la pregunta de, referida hacia la segunda y, con fines orientativos de la presente investigación, ¿Qué oportunidades configura la Corrupción a Gran Escala, desde un ejercicio deliberativo hacia su tipificación, para el fortalecimiento de la democracia pensada desde la base de la sociedad?

Buscando una explicación prematura, la concertación de la CAGE desde la base social, primero, fundamenta la legitimidad de lo que en una instancia legislativa sea tipificado como tal; y segundo, cierra la brecha existente entre ésta última y la comunidad civil; todo esto se enmarca en un ejercicio de continua retroalimentación y transparencia.

Trasversal al desarrollo, se identifican los siguientes objetivos: 1. Recopilar los elementos teóricos existentes alrededor de la Corrupción a Gran Escala, buscando enfatizar en el estado de la cuestión; 2. Examinar los elementos fundamentales de la Teoría de la acción comunicativa, entendida como imprescindible si a la deliberación en sociedad nos referimos; y, 3 . Plantear las oportunidades que la Corrupción a Gran Escala implica para el fortalecimiento de la democracia, toda vez que sea optado el camino de la disertación social en el que se amplíen los consensos y disensos sobre la materia, resultando, de este modo, el compendio de insumos que hagan las veces de idea-fuerza en estadios técnicos ineludibles para la construcción de la norma.

Referido esto, una primera sección tendrá incumbencia en los principales elementos de la citada Teoría, propuesta por el filósofo alemán Jürgen Habermas. Acto seguido, se planteará en la mayor medida posible el estado de la Corrupción a Gran Escala como noción, haciendo énfasis en literatura académica, así como en informes provenientes de organismos de alto nivel; vale la pena aclarar que, buscando una aproximación concreta, se inicia tomando la corrupción cual noción genérica para, de esta forma, exponerla desde su sentido privado del que asume varios elementos como parte integral de la categoría a estudiar. Tercero, se da paso a la mención de las oportunidades como aporte de la investigación en curso; y así, en cuarto lugar, se entregará una serie de conclusiones a manera de propuestas que ahonden en el carácter político de la Corrupción a Gran Escala, tomándose esta como un asunto público-privado de máxima importancia de acuerdo a sus efectos producidos en los Estados.

\section{METODOLOGÍA}

El desarrollo del presente estudio tendrá como herramienta de interpretación el método hermenéutico de las ciencias sociales. En él, resulta como elemento central la focalización sobre la materia a estudiar, entendiendo esta actividad como medio esencial para alcanzar su comprensión (Cárcamo, 2005). Usando la interpretación como medio se puede llegar a una comprensión total del fenómeno, mediante una operación que supone un conocimiento más profundo del objeto por medio de la desmembración, fundamentación y esclarecimiento de su estructura y relaciones contextuales (Mejía Quintana, 2014). En el momento de la aplicación se evidencia que la propia comprensión transforma lo comprendido; la hermenéutica devela aquí la realización de lo interpretado, demostrando su dualidad como saber teórico y su comportamiento como un fin práctico (Mejía Quintana, 2014).

Al plantearse una definición del método por sus partes, Ricoeur (2013) entiende por hermenéutica "[...] la teoría de las operaciones de comprensión en su relación con la interpretación de textos, en la que la palabra hermenéutica no significa otra cosa que la experiencia metódica de la interpretación" (p. 59). Ahora bien, acerca de la distancia trazada entre el comprender y el interpretar, el primero se arraiga como centro irreductible del segundo, abriendo la posibilidad de afirmar que la comprensión acoge los procedimientos explicativos desde una dinámica precedente, de acompañamiento y conclusión (Ricoeur, 2013). En últimas “[...] la comprensión contiene la explicación en la medida en que la explicación desarrolla la comprensión. Esta doble 
relación puede ser resumida en la expresión [...] explicar más para comprender mejor" (Ricoeur, 2013, p. 63).

Concorde a ello, el método hermenéutico, al centrar su elaboración y desarrollo desde la vivencia del o los individuos implicados en un escenario, asegura una mejor interacción y lectura entre los diversos sujetos de un contexto en específico; de allí que lo fundamental sea implementar diferentes utensilios que permitan la fundamentación y explicación que persisten en las relaciones del objeto en estudio.

Formulada esta herramienta, se tomará como punto de partida el estudio de la Teoría de la Acción Comunicativa y los elementos seleccionables alrededor de la materia tratada; acto seguido, se abordará la conceptualización de la CAGE, buscando descomponer por factores su implicación y comportamiento. Simultáneo a este ejercicio, serán puestos en diálogo conceptos y dinámicas observadas desde la Facticidad y Validez como obra de Jürgen Habermas en la que tiene desarrollo el paradigma discursivo del Derecho moderno. Contemplada ésta dinámica, se intentan recoger los conceptos e ideas suficientes para cubrir la intención principal del estudio.

\section{ELEMENTOS DE LA ACCIÓN COMUNICATIVA PARA UNA DEFINICIÓN DE LA CORRUPCIÓN A GRAN ESCALA DESDE LA BASE SOCIAL}

Adquirir los elementos correspondientes para una definición, desde la base social, sobre qué entender por CAGE, parece tarea que no descansa en una aleatoriedad teórica; en este sentido, se aborda la Acción Comunicativa, propuesta por el filósofo alemán Jürgen Habermas. Motivo de construir un ejercicio organizado, se iniciará por definir las dos acciones que Habermas encuentra imperantes en la edad moderna: las acciones estratégicas $y$, las acciones instrumentales, contrapuestas al modelo comunicativo de su Teoría y el carácter democrático del mismo, y en las que es adyacente el grueso de su crítica prevaleciente hacia las decisiones y procesos científico-administrativos. Sobre este juicio, reza Innenarity (1986) que "Habermas considera que en el fondo de toda la filosofía moderna subyace el intento de impedir una discusión racional en torno a las cuestiones públicas" (p. 240).

Ahora bien, de cara a la primera asignación, tanto las acciones estratégicas, como las acciones instrumentales, están contenidas en una categoría genérica representada bajo la noción de acciones, ellas, en palabras de Habermas (1987) significan "aquellas manifestaciones simbólicas en que el actor [...] entra en relación al menos con un mundo [...]" (p. 139). Entonces, es notorio que la acción en sí implica una interacción de al menos dos partes, motivo por el que todo resultado vinculado a una sola, quien obvie la existencia de un otro, es altamente cuestionable.

Tomando lo anterior como punto de partida, el límite impuesto tras una acción instrumental, según refiere Durango (2008), converge en que "[...] es insuficiente para lograr acuerdos perdurables socialmente, ya que la imposición que ejerce la lógica de medios y fines [...] es lo que genera la pérdida por el sentido político, la democracia y los derechos fundamentales" ( $p$. 48). A este parecer, entonces, se vinculan todas aquellas decisiones exentas de elaborarse tras un ejercicio de interpelación al otro, en donde prima una visión sustentada en el discurso de la eficacia y la eficiencia, por qué no, economicista.

Bajo este carácter impositivo, la acción estratégica "domina y promueve relaciones de dominación orientados al éxito y es controlada por el sistema administrativo y político [...]" (Durango, 2008, p. 48). Frente a esto, Innenarity (1986) genera una lectura sobre la misma, al afirmar que

La acción estratégica tiene, ciertamente la ventaja de que el significado puede ser establecido monológicamente, es decir sin esfuerzo hermenéutico. Pero la acción estratégica es un caso límite -no modélicode la acción social. Por eso, su aplicación indiscriminada conduce una inevitable desintegración (p. 242).

Considerando ambas una máxime expresión de la lectura elitista y exclusiva de la sociedad, predominante en las altas esferas del poder político y económico, el que en ellas se configure una noción de la CAGE, por lejos, divisa cierta cantidad de sesgos definitorios sobre el fenómeno, abstrayéndolo a un campo ideológico; $y$, de paso, a un discurso racionalista desde la eficiencia. De la misma forma, el carácter plural de su condición amerita que sea ampliamente discutido, elemento que no se encuentra en ninguna de las dos acciones mencionadas, ya que en estas "]...] el 
lenguaje es concebido unilateralmente, al tenerse solo en cuenta en cada uno de ellos alguno de los aspectos que el lenguaje ofrece" (Habermas, 1987, p. 137).

Si la idea hasta aquí promovida es que el definir la CAGE procure ser un ejercicio de "muchos", o al menos la base de su qué, resultaba entonces necesario enfatizar en ésta crítica, ya que, tanto en la acción estratégica, como en la acción instrumental, el planteamiento sobre la legitimidad y la autoridad solo puede ser conservada si ellas mismas se encuentran exentas de discusión (Innenarity, 1986). De continuar con éste último proceder, sería notoriamente comprometida la estabilidad del régimen democrático, toda vez que sus decisiones, especialmente hacia aquellos elementos de gran calado e importancia social, fueran ligados únicamente a un discurso racional de medios y fines.

Compartiendo una dimensión de la democracia en la que los elementos proyectados hacia el sistema político obedezcan a un fundamento sustentado en las demandas sociales, el principio discursivo, a través del cual estas últimas logren consolidarse, debe sobreponerse como método de optimización, en tanto brindan la legitimidad de aquello producido. Vivir en una democracia, ocasiona que la comunicación y la argumentación sean ejercicios fundamentales a la hora de aceptar los ideales que atan a los congregados, por ello "la racionalidad comunicativa, por lo tanto, no solo se refiere al mundo objetivo, [...] sino que incluye la intersubjetividad y la subjetividad, que componen conjuntamente el mundo de la vida." (Delgado, 2012 , p. 247), en este trato entre iguales, el optar por la democracia refleja una elección voluntaria y libre, adecuada a las situaciones que se aceptan tras la argumentación (Cortina, 1995b).

A este parecer, la Acción Comunicativa propuesta por Jürgen Habermas, tiene sus principales cimientos en el espacio de deliberación que se habilita por antonomasia en los Estados democráticos. Más allá de interpretarse como una tercera vía, contraria entonces a la acción estratégica e instrumental predominantes, la Acción Comunicativa yace cual proceso de entendimiento en el que la racionalidad humana entra en juego a través de relaciones intersubjetivas, buscando llegar a un acuerdo sea cual sea (Solares, 1996). Apegado a este escenario, la importancia del lenguaje descansa en dos situaciones: la primera, en tanto que amplía el horizonte del pluralismo, la tolerancia y la comprensión del otro; y la segunda, de cara a la posibilidad que trae en sí de alcanzar un fin consensuado hacia temas que requieren acciones comunes, haciendo énfasis en el enriquecimiento cognitivo de la deliberación (Vásquez, 1995).

Según lo visto, la deliberación habilitada en la democracia, difícilmente está exenta de una participación plural a través del lenguaje; en ella, los temas de suma importancia se ven sometidos a discusiones en las que convergen y divergen múltiples posturas sobre su fundamento. Como ejemplo de esto y a tientas de la materia que encuentra este estudio, la interpretación de la CAGE, antes de ser vinculada a regímenes técnicosadministrativos que influyan en su caracterización como hecho y delito, insta a ser habilitada desde la pluralidad como insumo legítimo, de lo luego superpuesto en el sistema social.

Continuando, el entrelazamiento de la Acción Comunicativa y el lenguaje, está respaldado en afirmaciones como las de Habermas (1987), una vez que en la primera

[...] hablantes y oyentes se refieren, desde el horizonte preinterpretado que su mundo de la vida representa, simultáneamente a algo en el mundo objetivo, en el mundo social y en el mundo subjetivo, para negociar definiciones de la situación que puedan ser compartidas por todos (p. 137).

No es de menos, entonces, que el lenguaje haga las veces de medio fundamental y a la vez de herramienta, en la interacción de puntos de vista de cada participante inmiscuido en el proceso, a los cuales se les dota o no de validez (Habermas, 1987). ¿Cómo se interpreta entonces la interacción de hablantes a través de la noción de Acción Comunicativa? Una primera respuesta logra encontrarse en la lectura que Vergara (2011) hace de Habermas al describir a la Acción como "[...] la interacción entre dos sujetos capaces de comunicarse lingüísticamente y de efectuar acciones para establecer una relación interpersonal" (p. 9). Adecuado a esto, los sujetos o participantes anteponen un saber intuitivo de interpretación general, frente a la tarea de lograr un acuerdo (Solares, 1996). 
De ser la Acción Comunicativa un operador o medio lingüístico en el que sea refleja la relación del actor (o actores) con el mundo (Habermas, 1987), la definición desde la base social de la CAGE permea los mecanismos democráticos en los que se conjuran múltiples voces y perspectivas hacia un fenómeno que, si bien resulta material, no goza de vida certera en el espacio del derecho y la norma; desde ello, el enfrentamiento de posturas ante la solución de un conflicto motivo de un tema público común, genera tras sí una "democratización" de la racionalidad, en donde el concepto de mundo de vida, propio de una tradición fenomenológica entra en juego (Gómez, 2003), gozando de un papel fundamental en la Acción Comunicativa y su desarrollo.

El interrogante entonces es volcado hacia la definición de mundo de vida, componente neurálgico de la Teoría expuesta. Procurando responder a ello, Gómez (2003) aborda a Habermas y contempla esta noción como “[...] una reserva culturalmente transmitida y lingüísticamente organizada de modelos interpretativos" (p. 64). En avanzada, Durango (2008) analiza que Habermas ve tras los mundos de vida, un entramado de acciones comunicativas, nutridas a partir de una multiplicidad de fuentes que hacen posible reflejar las tradiciones culturales, órdenes legítimos, etc.

La aglomeración de estas posturas, fortalecidas desde la experiencia del ser con su medio, fomentan una amplitud del tema a ser cuestionado. Con esto en mente, la Acción es tomada por un sistema abierto en donde los participantes interactúan y proveen a tal estadio de deliberación una fuerte carga de significados, producto de sus experiencias y trasfondo que el entorno inmediato les provee; tal afirmación cobra forma bajo el concepto de mundo de la vida. En éste último estadio, el de la interacción subjetiva, el lenguaje persiste como un medioherramienta a través del cual los involucrados logran brindar pretensiones de validez a situaciones comunes y las definiciones con las que intentan caracterizarlas.

Desde la Acción Comunicativa y como ya fue expreso, su carácter basado en la pluralidad y puesta en marcha de un mundo de perspectivas, la búsqueda de un espacio democrático en el que se defina desde el amplio social el fenómeno de la CAGE genera consigo una primera oportunidad para la democracia, que, no sin antes advertir será desarrollada en la parte final de este estudio, se ubica en el aspecto legitimador que Habermas brinda a la deliberación. Al respecto, Zoffoli (2010) advierte que "[...] es absolutamente necesario que las normas resulten aceptables a los ojos de quienes serán sus destinatarios, si se quiere que ellas sean no sólo normalmente válidas, sino también socialmente creíbles y por ende políticamente practicables" (p. 73). En consecuencia, el derecho (desde la producción de normas), debe ser tomado como un espacio en sí presto a los acuerdos que se tomen desde el campo social.

En sentido bidireccional, Habermas (1998) determina que "El proceso de producción de normas constituye por tanto en el sistema jurídico el auténtico lugar de la integración social" (p. 94). De esta forma, al introyectarse lo social en lo legal, la primera despliega ante la segunda un componente de legitimidad, puesto que, las herramientas de la última, conciben la maximización del bienestar en la sociedad.

Ahora bien, de esto se despliega una legitimidad discursiva del Derecho, la que, en palabras de Mejía Quintana (1997) y dando seguimiento a la obra de Habermas, se formula a partir de:

El giro que representa su propuesta de un paradigma discursivo del derecho constituye el reconocimiento de los procedimientos jurídicos, en tanto sus contenidos garanticen la multiplicidad de perspectivas del mundo de la vida, puede ser el elemento más eficaz para rehacer el lazo social desintegrado desde una posición dialogal que supere los límites del paradigma monológico de la modernidad ( $\mathrm{p}$. 33).

Postulado el último aparte, se dista de aplazar la creación de leyes desde un modelo diagnósticodiscusión-resultado, ni mucho menos se intenta obviar la rigidez de su aspecto técnico. $\mathrm{Al}$ contrario, entendiendo que es en las instancias legislativas donde se halla uno de los mayores fundamentos de la democracia, se observan tras ella misma y sus resultados los componentes que brindan legitimidad a un sistema. A esta razón, la construcción de qué entender por CAGE, antes de someterse al imperio técnico de las corporaciones legislativas, debe verse filtrada tras la deliberación 
en donde confluyen, según fue mencionado, una diversidad amplia de experiencias y puntos de vista, que alimentan el debate y dotan de legitimidad las decisiones producto de lo allí acordado.

En aras de cumplir con la estructura ya formulada, el siguiente apartado intentará recopilar la mayor cantidad de referencias que, desde la literatura académica, se han realizado acerca del concepto de CAGE, ejercicio que despierta, más allá de un enriquecimiento sobre el tema, las bases de una segunda oportunidad visible para con el fortalecimiento de la democracia.

\section{UNA MIRADA DE LA CORRUPCIÓN A GRAN ESCALA DESDE LA ACADEMIA}

El abordaje de la Corrupción, así, desde su sentido genérico, descansa en un compilado de autores, teóricos y analistas que, tras años, han intentado definirla. Motivo de este proceso, actualmente se tiene claro entre tanto discernimiento el que el acto corrupto no se desliga de la consumación del interés propio sobre el colectivo, así como que sus esferas de incidencia van más allá de lo público (pensando en el sector oficial), alcanzando límites de lo privado e, incluso, lo comportamental.

En este sentido aclaratorio sobre los patrones de conducta y en el intento por delimitar las partes que toman lugar en todo acto corrupto, independiente al adjetivo que la acompañe (pública, privada, A Gran Escala, como formula el estudio) Vásquez (2007) establece:

En los fenómenos de corrupción es necesaria la presencia de una autoridad o de un decisor, entendiendo por tal todo agente con capacidad para tomar decisiones y cuya actividad esté sujeta a determinado tipos de deberes. Éstos se adquieren a través de un acto voluntario por el que alguien acepta asumir un papel dentro del sistema de reglas (p. 209)

De acontecer el acto corrupto, se establece un comportamiento desleal hacia el sistema de reglas que voluntariamente el o los actores han asumido; de esto último se deriva su categorización como dinámica participativa, cuyo objetivo es obtener un beneficio extraposicional (Vásquez, 2007). Bajo esta mirada incisiva, previo a la identificación de cuán abordada ha sido la CAGE en la academia, las ideas de Elliott (1997) alrededor de lo definido como Petty Corruption, o "Pequeña Corrupción" y Grand Corruption o "Gran Corrupción", resultan imprescindibles al momento de tipificar a la Corrupción Privada, ésta, como categoría frecuentemente aislada de la que poca consideración se tiene hacia sus efectos en lo público.

Sobre lo referido a la "Pequeña Corrupción", esta tiene lugar cuando "actores privados interactúan con actores gubernamentales con cargos de no-elección, particularmente de bajo nivel [...] en ésta se tranzan impuestos, regulaciones, licencias requeridas, para el mayor beneficio del actor privado"1 (Elliot, 1997, p. 178). De esto, entonces que sean develadas conductas las cuales, en muchas ocasiones, tengan como resultado una desviación y efecto sobre el sistema de mercado y la competitividad de las empresas, tanto beneficiadas como antagónicas. Por su parte, la "Gran Corrupción" implica una interacción entre partes del alto nivel de gobierno, líderes políticos, burócratas y el sector privado ${ }^{2}$ (Elliot, 1997), la cual genera, igualmente, distorsiones sobre la estabilidad económica de un país.

Tomados estos elementos, la corrupción privada alberga dos posturas: una de ellas, expuesta en Nieto Martín (2002), cuya premisa da lectura de esta tipología con base a los constreñimientos y actos por encima de un conjunto de reglas, en el entorno laboral, buscando una ventaja sobre el vacío legal o el aumento de capacidades competitivas por las empresas; para esto, acude al caso de la legislación alemana, francesa y nórdica, tomando como punto de partida el hecho de que al interior de un mismo marco de cooperación entre países, el establecimiento de la conducta punible cambia y, por lo tanto, ampliando la idea de cuán difusa es la corrupción en sí. La segunda, pero no menos importante, se establece en Amundsen (1999) en donde a la connotación "privada" de la corrupción, se le otorga un carácter sociológico, cuyo fin último es la colectivización de un beneficio obtenido por fuera de los límites establecidos. Según esta postura,

Sin embargo, la corrupción es quizá algo "colectivo". Primero, porque la corrupción

1. Traducción propia.

2. Traducción propia. 
tiene un efecto económico sustancial en términos agregados y también porque ella quizá sea una forma deliberada para la extracción de recursos benéficos para un grupo más grande. [...] A medida que la corrupción se expande, su aceptación social también. Cuando es generalmente entendido que hay un clima de corrupción, muchas más personas la tomarán como algo inevitable. ${ }^{3}$ (Amundsen, 1999, pp. 4-5).

De cara a estas consideraciones, el arraigamiento de la corrupción a únicamente la dimensión pública del fenómeno, más allá de significar una limitación ante los estudios que la abarcan, precisa un asunto clave de cara a su tipificación como conducta punible, en tanto que su diagnóstico es altamente volátil prestándose como elemento tratado solo desde lo político y como una tarea irrealizable en lo que compete su mitigación.

Buscando discernir sobre las múltiples dimensiones que adquiere el fenómeno, tal como fue mostrado, la revisión de cuán estudiada ha sido la CAGE a través de aportes académicos, da ejemplo de los múltiples matices con los que, desde la teoría, se ha abordado el fenómeno. Resulta importante aclarar que, como se ha venido haciendo, su interpretación desde la dimensión normativa ha sido difusa, condición a través de la cual, al limitar el carácter punible de la conducta, se generan pérdidas de legitimidad en el sistema político, especialmente hacia sus instancias legislativas y judiciales; este punto comporta vital advertencia, puesto que, como se dejará en claro, la concepción académica de la CAGE refleja la imperiosidad del daño que esta ocasiona en el sistema.

En primer lugar, onda decir que la tarea no resulta sencilla, toda vez que "La propia naturaleza de la corrupción a gran escala implica que sea difícil abordarla eficazmente" (Martini, 2015, p. 2). A pesar de esto, ante los casos más graves de corrupción y la lucha por evitar la impunidad (además de sus efectos indirectos a largo plazo), existe un consenso y trabajo mancomunado a nivel internacional; esto teniendo en cuenta que no pueden emprenderse acciones legales contra una conducta criminal, sin saber en qué consiste el objeto en sí (García, 2016) no obstante, la

\footnotetext{
3. Traducción propia.
}

tipificación de la misma logra escapar a un mero protocolo legal, convergiendo un comportamiento de extensa reprobación social (Soriano, 2011).

Frente a estas afirmaciones, se genera una condición material del fenómeno, que repercute en la experiencia de vida de quienes la padecen, sea de manera directa o no y, que nutre su lenguaje a través de una postura que se guarde frente a él.

Con esto en mente, una primera definición es aportada por Transparencia Internacional (2009) para quien "La corrupción a gran escala consiste en actos cometidos en los niveles más altos del gobierno que involucran la distorsión de políticas o de funciones centrales del Estado, y que permiten a los líderes beneficiarse a expensas del bien común" (p. 23). A su vez, García (2016) desagrega la noción por partes y la limita a casos en los que

a) involucran a enormes sumas de dinero [...]; b) son ejecutados, auspiciados, dirigidos o tolerados por personas con altas cotas de poder o sobre el gobierno [...]; c) no constituyen comportamientos aislados, sino un conjunto de actos planificados que se insertan en el propio funcionamiento del país o región afectada [...]; d) el perjuicio causado no es solo económico, sino que afecta también a los derechos fundamentales y libertades públicas del conjunto de los ciudadanos [...]; e) la relación de los corruptos con las altas esferas de poder y el carácter planificado de sus actos les permiten eludir la acción de la justicia, ya sea impidiendo coactivamente su actuación, ya sea premiando su tolerancia [...] (p. 65).

Motivo de estos elementos, cobijados por lo que teóricamente se entiende por CAGE, se visibilizan tres principales escenarios en los que tal conducta incide de sobremanera en el sistema político de un país: 1 . La sistematicidad que la conducta posee como punto de inicio, involucrando así (y generando mayor dificultad para ser contrarrestada) la cantidad de nodos que deben abordarse para su mitigación, además de la heterogeneidad jurídica al tener un carácter regional o, incluso, internacional. 2. La CAGE tiene perjuicio sobre los derechos fundamentales de la población, sea por una omisión en las funciones del Estado, sea porque sus efectos, más allá del carácter económico, se arraigan en el sistema 
bajo una dinámica contraproducente, coartando la capacidad de agencia, el progreso individual y el colectivo. 3. La estrecha relación que guarda la CAGE con las esferas del poder, generando un malestar de impunidad hacia las instituciones estatales y su capacidad de recepción-respuesta.

Uno a uno, entonces, muestra la necesidad de realizar una aproximación teórica hacia el fenómeno social y jurídico que refleja la CAGE, desde la base social; y, proponiendo el debate en pro del consenso al respecto. Al tiempo que es resaltado cómo los mundos de la vida de los integrados en una comunidad se comprometen y guardan, desde un carácter material, las experiencias frente al devenir. De no presentarse lo anterior, la justicia como fundamento principal del Estado Social de Derecho, sobre el cual apuesta su actuar si procura ser legítimo (Cortina, 1995a) es una de las principales virtudes y funciones que se asumen como frágiles.

Bajo este mismo hilo de ideas, el que se limiten los derechos fundamentales desde el accionar del Estado (o peor aún, desde su omisión), va en contravía de un principio mediante el que, al ser posibles, legitiman un orden jurídico y político (Durango, 2006). Así, se da una lectura de los mismos como garantía que debe conceder el Estado en procura de mantener la integración social, a partir de sus funciones y la eficacia de las mismas.

Por último, un componente que agrega Ceballos (2018) al cuerpo de las definiciones resaltadas, es que, además de identificar entre los involucrados a miembros de las ramas del poder, incluye también elementos del sector privado, situación que agrava la conducta, dada la imperiosidad del mercado como lógica sobre la cual los distintos gobiernos toman decisiones, dejando latente una relación de utilidad en términos particulares. En este orden, Pino et al (2020) entiende la CAGE como

aquél acto corrupto desarrollado por agentes de alto nivel, activos o con participación latente en el sistema político, lugar donde no interesa su rol -sea funcionario público, político o actor privado-, a través del cual se busque alcanzar un beneficio económico, de influencias o favores, y que, al alterar el orden de las reglas en el Estado de Derecho y la democracia, no permite una óptima distribución de los recursos, los servicios y las oportunidades como garantías obligatorias a brindar por el Estado (Párr. 47).

En términos prácticos, la alteración de las reglas de juego y el debilitamiento de la democracia en un sentido material y funcional, resultan señalados como aspectos definitorios de la CAGE, implicando así una afectación de las instituciones, así como de los derechos fundamentales con los que cuenta la población de un país, dejando en entredicho la legitimidad funcional $^{4}$ del Estado. Bajo estas condiciones, la ilegitimidad material del Estado "se refiere al hecho de que las estructuras que edifican el Estado no están cumpliendo con los cometidos por la dogmática constitucional" (Rúa Delgado, 2013, p. 99). Alrededor de ésta dinámica y según los actores establecidos como parte del desarrollo de la CAGE, las instituciones se trastocan al punto de traer sobre el estudio, obligatoriamente, los conceptos de Captura del Estado (CdE) o Reconfiguración Cooptada del Estado (RCdE).

Haciendo énfasis en estos dos, Garay, SalcedoAlbarán, León-Beltrán y Guerrero (2008) asimilan la CdE como una variante de la CAGE, en la que específicamente "se debilita la estructura económica de un país porque distorsiona la formulación de leyes, normas, decretos, reglas y regulaciones" (p. 15). A este parecer, la CdE se interpreta bajo una relación Estado-actores privados, por medio de la cual se busque un beneficio, tranzadas facilidades hacia un sector económico o del mercado, desde los compromisos jurídicos y legales.

En este mismo sentido, planteada más como una extensión de la CdE que una contraparte, la RCdE desdibuja un principio "legal" del actor particular involucrado, en tanto que, determinado el concepto desde el caso colombiano, quien hace sus veces, ya en sí pertenece o ha pertenecido a una organización criminal; la postura en Rúa Delgado (2013) sobre la materia, indica que el proceder de esta categoría se realiza desde el mismo interior del Estado, abarcando plazas y lugares de importancia estratégica en la elaboración de las reglas de juego.

4. De acuerdo con Jurado (2000), la legitimidad funcional toma forma en el momento en que un gobierno logra cumplir, a través de un desempeño eficiente, las tareas que tiene asignadas, siendo estas acordes a las aspiraciones de la población. 
Mencionado lo anterior, si una primera oportunidad se devela en el entrelazamiento de la CAGE y la deliberación, como vehículo para fortalecer democráticamente las instituciones, en tanto lo que se decida frente a la caracterización del fenómeno es, teóricamente hablando, una convergencia de perspectivas en los espacios de participación, la segunda oportunidad yace entonces bajo los límites sobre lo material, en tanto se adopta una posición de mitigar los impactos de la CAGE, ya sea a través de instancias judiciales, o a través de herramientas políticas. Es sobre esto que encuentra fundamento el quéhacer del Estado y, al impactar sobre el sistema, se genera una retribución en carácter de legitimidad.

Habilitado este compendio de definiciones académicas, tanto de la corrupción en su categoría general, pasando por la corrupción privada como uno de las categorías inevitables para el abordaje de la CAGE, sus actores y los comportamientos que en ella tienen lugar, así como el trato de la CAGE y la suerte de concepto paraguas que obtiene desde su noción, queda entonces por resolver, tal como se mostrará en el siguiente apartado, la especificidad de las oportunidades que, en este estudio, hemos resuelto, encuentra la democracia y su fortalecimiento, tras el fenómeno de la CAGE.

\section{OPORTUNIDADES DESDE LA CORRUPCIÓN A GRAN ESCALA PARA EL FORTALECIMIENTO DE LA DEMOCRACIA}

Los hallazgos que amerita el hilo conductor de la argumentación hasta ahora planteada, giran en torno a dos ejes fundamentales: por un lado, se establece que la tipificación del fenómeno de la CAGE como delito, buscando otorgarle existencia en el compendio normativo y/o penal, debe estar sujeta a una primera fase de deliberación social cuyo insumo principal sería, entonces, la validez de la norma creada en procura de identificar y condenar al mismo. Por otro lado, el que se haya caracterizado la conducta implica la adquisición de una postura, tanto material como legal al ahora denominado delito, encontrando en los diseños resueltos con el fin de mitigarlo, una finalidad de constreñir el deterioro que él ocasione al sistema político, siendo el grueso social los principales destinatarios de lo que actualmente deriva de tal fenómeno.

En este orden, una primera oportunidad tal cual había sido mencionada, es la validez de la norma con la que se tipifique la CAGE, sustentada en el espectro deliberativo que tiene lugar en el Estado democrático. A entenderse, se hipotiza en que, la participación respecto a qué conceptualización jurídica reciba la CAGE y su inmersión como delito al sistema normativo, debe antes acontecer en un estadio de interlocución y, así, dotarle de legitimidad a partir de una interacción de los mundos de vida. En tal sentido, Vásquez (1995) analizando la Acción propuesta por Habermas, expone que "Para la democracia solo pueden reclamar legitimidad las leyes que al establecerse legalmente en un proceso discursivo pueden encontrar acuerdo entre los asociados" ( $p$. 59).

Esta consecución de validez, reposa en primer lugar sobre los elementos que entrega la Acción Comunicativa y la manera en que se conforman discursos a través de la participación plural, con incidencia en la toma de decisiones; con base a ello, la categoría de principio discursivo, fundamental para la creación de normas, no debe excluirse de este primer escenario. Según qué entender por éste principio, Durango (2006) lo interpreta como un "argumento racional que acoge concepciones pluralistas, pues al tener la capacidad de articular variados intereses particulares, éste se convierte en sustento teórico, a la vez que funcional como mecanismo reflexivo en la construcción y producción de normas legales y legítimas" (p. 19). Así mismo, en una dinámica organizada, "el principio D se limita a explicitar la justificación imparcial de las normas de acción en general" (Martín, 2005, pp. 13-14). Tal cual se entiende, la conformación de la norma, en la motivación de orientarse frente a un asunto público y de gran magnitud, debe hallar su legitimidad a través del consenso en el que las experiencias de los participantes entran en juego.

Tal ápice de participación, como condición sin equa non se puede otorgar validez a una norma, es característica fundamental de los regímenes democráticos. Ante esta afirmación generada desde el derecho, Mejía Quintana (1997) señala que

relaciona las dos caras de esta tensión en una interrelación que hace del derecho, por una parte, en tanto hecho social, forzosamente coercitivo a fin de garantizar los derechos ciudadanos y, por otra, en tanto procedimiento para conformar la ley, abierto 
a una racionalidad discursiva legitimatoria, democráticamente organizada (p. 36).

Esta racionalidad discursiva, confluye en el límite de acciones que se plantean desde el derecho, el cual a su vez debe otorgar el marco de participación mediante el cual, pasa a ser validado a través de la actitud argumentativa de los involucrados en la producción de normas (Cuchumbé, 2004).

Cumplida esta primera fase y sustentada en la idea de que el acercamiento a la realidad social no tiene mejor forma que a través de los espacios deliberativos, públicos en su naturaleza y habilitados como escenarios legales para la discusión y posterior toma de decisiones (Domínguez, 2013), la segunda oportunidad se relaciona con el carácter material del delito, ya que, al ser identificado oportunamente, tras un ejercicio deliberativo plural y con influencia en la decisión que lo tipifica y le da vida en el sistema legal, el qué-hacer del Estado divisa una postura de cómo actuar y mitigar la conducta. Así, desde la incidencia de sus funciones, el valor con el que se legitime tendrá mayores oportunidades de ser medido.

En este sentido, la búsqueda por definir socialmente la CAGE, atraviesa un instante democrático mediante el cual, como resultado, se reformula la manera en que el sistema político sortea las dificultades que tal fenómeno proyecta sobre su qué-hacer cotidiano; luego entonces, de no buscarse ello

el distanciamiento de las instituciones sociales respecto a las demandas de la sociedad, y la legalidad de la propiedad, constituyen el mayor cuestionamiento a la legitimidad del Estado, la democracia y el derecho, conformando la situación de crisis en las sociedades actuales (Ortega, 2014, p. 106).

La puesta en escena de aquellas herramientas concebidas para reducir el impacto de la CAGE, no sólo deben pensarse como una postura del sistema ante el delito, sino también en la búsqueda que este mantiene sobre la integración y cohesión social, al entender las enormes brechas que tal fenómeno ocasiona sobre los derechos fundamentales y la capacidad de agencia de las personas. De esta misma forma, el fenómeno de la
CAGE acentúa el qué-hacer omisivo del Estado en tanto que imposibilita la concreción de funciones que busquen un bienestar general, postergando una connotación negativa del poder que ejerce este último sobre los integrados y haciendo material esta forma como la única mediante la cual toma parte. Según ello, el rol del derecho debe concebirse como un elemento de mediación social, en donde hechos y normas interactúan (Mejía Quintana, 1997).

La dinámica entonces, de no ser desarrollada, haría que el círculo de retroalimentación entre Derecho-Racionalidad Comunicativa se vería constreñido, aún más, al imperio de lo legal en desconocimiento de la realidad social; la legitimidad, entonces, implicaría más una cuestión de forma -sobre la base procedimentaly bajo una intención meramente instrumental, desplazando la realidad del fenómeno como un asunto de fondo y con potencial elaboración desde la interlocución y el diálogo extensivo.

Una visión mecanicista de ambas oportunidades, para el fortalecimiento de la democracia, es sintetizada en la figura 1, según es mostrada a continuación.

En aras de realizar un análisis sobre el esquema anterior, es fundamental precisar que consta de tres partes, donde, la primera y la última guardan efectos previos y a posteriori, respectivamente. Acerca de los efectos que la CAGE arroja sobre la sociedad o construye, se observa una fractura de la legitimidad funcional y formal del Estado, dado el síntoma difundido sobre la inoperancia y la cooptación del mismo por parte de intereses particulares. Alrededor de esta premisa, se construye un segundo efecto, en el que el Estado alberga únicamente un discurso de unión hacia la élite, desplazando las acciones que busquen un sentido benéfico colectivo. Como resultado, igualmente, se postula la aparición de actores alternativos para la satisfacción de intereses sociales, cuya legitimidad puede tomar tintes violentos y/o de explotación.

A esta razón, los impactos de los efectos materiales producidos por la CAGE se interponen y resultan un input trazable en los mundos de vida de la base social, arrojados al exterior bajo la forma de un discurso en cual, frecuentemente, congrega lo reclamado ante el Estado como esa entidad garantista de bienes y servicios. 
Figura 1. Formulación de insumos desde la base social para la definición de la CAGE

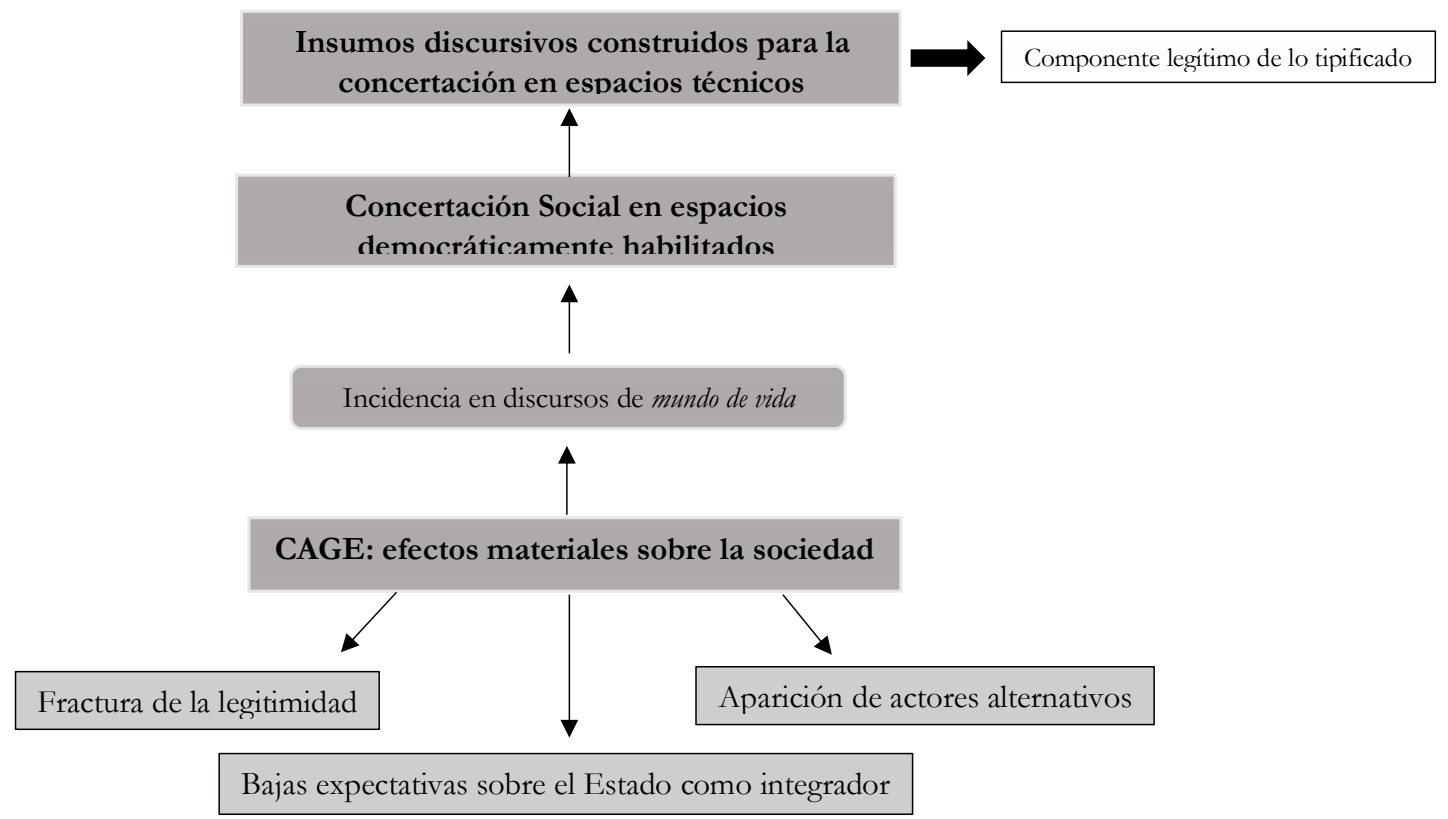

Fuente: Elaboración propia

De esta manera, una vez se habiliten espacios que respeten la disertación de los mundos de vida alrededor del fenómeno en estudio, se recopilan los disensos y consensos que existan, buscando democratizar los insumos que, posteriormente, comporten el fundamento legítimo de la conducta tipificada. Aquí se despliegan los elementos analizados desde la postura habermasiana sobre la validez y legitimidad de las normas, según prospere en espacios participativos. El componente legítimo de lo acertado, en un estadio técnico del derecho y la creación de normas, reposa en su previa asimilación desde la base social como una entidad que se enfoca en distintas aristas y perspectivas con las que alimentar el debate.

\section{CONCLUSIONES Y RECOMENDACIONES}

Resulta inevitable dejar de pensar cuántas crisis afronta el Estado Social de Derecho, acudiendo a su carácter garantista y legitimado en conjuntos de normas fundamentales cuyas disposiciones orientan su qué-hacer. A merced de los obstáculos planteados hacia la materialización de sus funciones, son cada vez más las dudas que se plantean alrededor del sistema político, especialmente hacia aquellos derechos otorgados mediante su acción continua y dimensionados a través de bienes y servicios.

Tal y como se anotó, la Corrupción desde una definición genérica presenta diversas limitaciones a la hora de examinar la multiplicidad de fenómenos que, a su nombre, parecen inundar la cotidianidad de la acción administrativa; desde la Corrupción enfocada en lo público, pasando por la categorizada como "privada", todas ellas delimitan un fenómeno en el que el usufructo de los particulares a merced del beneficio común es notable. De cara a lo mencionado, la Corrupción a Gran Escala representa un desafío aún mayor, no solamente desde sus efectos materiales, sino desde el desconocimiento de qué considerar como tal y las implicaciones que ello trae para su tipificación en el marco del derecho.

A esta razón, sin reducir la complejidad de la categoría, la base social y sus discursos en los que salen a flote los mundos de vida construidos alrededor de los efectos materiales de la Corrupción a Gran Escala, comportan un 
elemento fundamental de cara a la amplificación y discernimiento democrático sobre la conducta, otorgando un espacio participativo alrededor de aquello dañino para la vida en comunidad, cuya principal forma material se logra divisar en diferentes escenarios, tales como la Captura del Estado, la Reconfiguración Cooptada del Estado -ambas, estadios avanzados de Corrupción a Gran Escala- y la omisión sobre el desarrollo de los derechos fundamentales. En un paréntesis, se destaca la complejidad dada la no posibilidad de desconocer el factor técnico para la creación de normas, así como la interpretación sobre los límites difusos que hacen ser, y luego no, al delito en cuestión.

Bajo esta idea, la trazabilidad de qué entender por Corrupción a Gran Escala retoma elementos propios de la Acción Comunicativa dispuesta por Jürgen Habermas, toda vez que se dota de racionalidad al lenguaje y, como resultado, un aspecto legítimo de la norma según lo que se concierte como tal: la participación desde la base social configura, además de un espacio legítimo, un entorno democrático en el que se profieran elementos de peso sobre la Corrupción a Gran Escala al momento de ser tipificada como delito en estadios técnico-administrativos.

Con bases a estas premisas, una primera oportunidad para el fortalecimiento de la democracia desde la Corrupción a Gran Escala se devela en la instancia participativa, cuyo desarrollo se enfoque en la pluralidad de ideas $\mathrm{y}$ opiniones que, en un ejercicio deliberativo organizado, recopilen las aproximaciones de los integrantes de la base social como interlocutores principales y con argumentos desde sus mundos de vida. A pesar de esto, son varias las limitantes que podrían acontecer en el proceso, considerando la pobreza, la desigualdad social y la suspicacia ante las vías legítimas de concertación, como producto mismo del Estado que, en últimas, ha omitido su papel garantista.

Una segunda oportunidad que tiene el fortalecimiento de la democracia desde la concertación social acerca de la Corrupción a Gran Escala, se ubica en una dimensión material, dado el argumento en el que esta misma corrupción socava las capacidades del Estado, alejándolo funcionalmente de la población que ha instado por resguardar. Si bien el proceso no resulta sencillo, la identificación de los insumos establecidos desde la generalidad hacia la definición posterior de la conducta como un crimen, habilita que esta última entidad posea una cuantificación en la que base su creación de herramientas para contenerlo y mitigarlo.

De esta forma, la puesta en marcha cabalmente de las funciones del Estado, ingresarían cual suerte de retroalimentación en forma de legitimidad sobre el sistema, estableciendo patrones de aceptación común ante el mismo fenómeno que prescribe la existencia del Estado y su facultad de coerción.

Si bien puede resultar un ejercicio ideal y paquidérmico en su desarrollo, el compromiso de la academia bajo el mundo de la teoría no puede desligarse de una lectura acerca de las nuevas realidades sociales y cómo, en ellas mismas, persisten una serie de discursos constreñidos bajo la racionalidad que tiene forma en las altas esferas y, en muchos de los casos, generan una visión única del problema, desentendiendo las dinámicas y hechos materiales que tienen lugar.

\section{BIBLIOGRAFÍA}

- Amundsen,I.(1999). "Political Corruption: An Introduction to the Issues". CMI, 1-32. Disponible en https://cutt.ly/ZcJVIqk

- Bueno, W., y Manzur, J. C. (2012). "Estado Social de Derecho: Consideraciones sobre su trayectoria histórica en Colombia a partir de 1991". Cuestiones Políticas,
№48(28), 51-77. Disponible en https:// cutt.ly/QftXIA3

- Cárcamo, H. (2005). "Hermenéutica y análisis cualitativo". Revista de epistemología de las ciencias sociales Cinta de Moebio, (23), 204-216. Disponible en https://cutt.ly/zftXPuU 
- Ceballos, A. (2018). "Colombia: de la corrupción común a la corrupción a gran escala". Revista Nova et Vetera, №37(4). Disponible en https://cutt.ly/3ftXAvd.

- Cortina, A. (1995a). "Presupuestos morales del Estado Social de Derecho". En Motta, C. (Comp.) Ética y Conflicto, Lecturas para una transición democrática. Bogotá: Tercer Mundo, pp. 185-206.

- Cortina, A. (1995b). Razón comunicativa y responsabilidad solidaria. Salamanca: Ediciones Sígueme.

- Cuchumbé, Nelson. (2004). “Acción comunicativa, Derecho y Organización social: el concepto de derecho en Habermas". Criterio Jurídico (4), 109-122. Disponible en https://cutt.ly/sftXDTp

- Delgado, G. (2012). "Los aportes de la teoría de la acción comunicativa y sus conceptos a una contabilidad para el entendimiento y la integración de la sociedad". Universitas Humanística, (74), 227-263. Disponible en https://cutt.ly/ LftXGgB

- Dominguez, H. (2013). "Democracia deliberativa en Jürgen Habermas". Analecta Política, Vol.4 (5), 301-326. Disponible en https://cutt.ly/7ftXJim

- Durango, G. (2006). "El principio discursivo y los derechos fundamentales en la teoría habermasiana". Opinión Jurídica, Vol.5 (9), 13-32. Disponible en https://cutt.ly/xftXLfp

- Durango, G. (2008). "Análisis, Crítica y Legitimidad del Derecho Moderno desde la Perspectiva de la Acción Comunicativa Habermasiana". Jurídicas, Vol.5 (2), 45-69. Disponible en https://cutt.ly/7ftXZBN

- Elliot, A. K. (1997). "Corruption as an International Policy Problem: Overview and Recommendations". En Elliot, A. K. (Ed) Corruption and the Global Economy. pp. 175-233. Institute for International Economics. Disponible en https://cutt. ly/tcJVHN4
- Garay, L., Salcedo-Albarán, E., LeónBeltrán, I., Guerrero, B. (2008). La Captura y Reconfiguración Cooptada del Estado en Colombia. Bogotá: Fundación Método, Fundación Avina y Transparencia por Colombia.

- García, E. (2016). “Gran corrupción: estrategias para evitar su impunidad internacional". Revista Nuevo Foro Penal, Vol.12 (87). 60-98. Disponible en https:// cutt.ly/oftXCJH

- Gómez-Díaz,Á.(2003). “Ética y corrupción. Lo público y la democracia”. Convergencia, Revista de Ciencias Sociales, 10 (31), pp.: 141-151. Disponible en https://cutt.ly/ gfySFs3

- Gómez, M. (2003). “Lenguaje y Acción en la Teoría de la Acción Comunicativa de Jürgen Habermas". Revista de Estudios Políticos (Nueva Época), (131), pp.: 31-70. Disponible en https://cutt.ly/oftXNpm

- Guitart, E. (2011). "Una interpretación de la psicología cultural: aplicaciones prácticas y principios teóricos". Suma psicológica, 18 (2), 65-88. Disponible en https://cutt.ly/NftXM4S

- Habermas, J. (1987). Teoría de la acción comunicativa, I. Racionalidad de la acción y racionalización social. Madrid. Taurus.

- Habermas, J. (1998). Facticidad y validez, Sobre el derecho y el Estado democrático de derecho en términos de teoría del discurso. Madrid. Editorial Trotta.

- Innenarity, D. (1986). "La teoría discursiva de la legitimidad de Jürgen Habermas". Persona y derecho: Revista de fundamentación de las Instituciones Jurídicas y de Derechos Humanos, (14), 233-278.

- Jurado, R. (2000). "Fundamentos de la legitimidad". Estudios Políticos, (24), 129-153. Disponible en https://cutt.ly/ eftX9p9

- Martín, F. (2005). “La razón práctica y el principio del discurso en Jürgen 
Habermas". Revista de Filosofía, Vol.23 (50), pp.: 1-38. Disponible en https://cutt. ly/qftX4KW

- Martini, M. (2015). “Combatir la corrupción a gran escala: desafíos y éxitos". Transparency International. Disponible en https://cutt.ly/zftX77f

- Mejía Q., O. (1997). “La Teoría del Derecho y la Democracia en Jürgen Habermas: En torno a Faktizitat und Geltung". Ideas y Valores, (103), pp.: 32- 52. Disponible en https://cutt.ly/aftCefA

- Mejía Q., O. (2014). "Elementos para una hermenéutica crítica: una introducción al problema del método en las ciencias sociales y el derecho". Pensamiento Jurídico, (39), pp.: 15-53. Disponible en https://cutt.ly/TftX6eP

- Michelini, D. (2015). "Deliberación. Un concepto clave en la teoría de la democracia deliberativa de Jürgen Habermas". Estudio de Filosofía Práctica e Historia de las Ideas, 17 (1), pp.: 59-67. Disponible en https://cutt.ly/hjdC9F2

- Nieto M., A. (2002, 12-13 de abril). "La corrupción en el sector privado (reflexiones desde el ordenamiento español a la luz del Derecho comparado)". Ponencia presentada al Convengo di studi su La corruzione tra privati: esperienze comparathistiche e prospettive di reforma, Milán.

- Ortega, J. (2014). "Crisis en la legitimidad del Estado, del derecho y de la democracia liberal". Pensamiento Jurídico, (41), pp.: 105-126. Disponible en https://cutt.ly/ QftX6JJ

- Pino, L; Medrano, A; Duarte, T; Mambuscay, J. “La corrupción a gran escala no tipificada en el ordenamiento colombiano: análisis desde los escenarios jurídicos y categorías de corrupción". Opinión Jurídica, 20(42) (Julio-diciembre de 2021), pp.: 143-166

- Pont, V.; Arango, M. P. (2017). "Sobre la corrupción en Colombia: Marco
Conceptual, diagnóstico y propuestas de política". Cuadernos FEDESARROLLO, (56). Disponible en https://cutt.ly/8ftCwan

- Ricoeur, P. (2013). "La hermenéutica y el método de las ciencias sociales". Cuadernos de Filosofía Latinoamericana, 34, (109), 57-70. Disponible en https:// cutt.ly/mftCtr]

- Rúa D., C. (2013). "La legitimidad en el ejercicio del poder político en el Estado Social de Derecho. Una revisión desde el caso colombiano". Ius et Praxis, 19(2), pp.: 85-121.

- Solares, B. (1996). “La teoría de la acción comunicativa de Jürgen Habermas: tres complejos temáticos". Revista mexicana de ciencias políticas y sociales, 163, (41), 9-33. Disponible en https://cutt.ly/TftCyuE

- Soriano, R. (2011). "La corrupción política: tipos, causas y remedios”. En Soriano, R., y G. Trocello (Coord.). Calidad Democrática e Instituciones Políticas, pp. 39-65, Sevilla: Acongagua Libro.

- Transparencia Internacional. (2009). Guía de lenguaje claro sobre la lucha contra la corrupción. pp. 1-57. Disponible en https://cutt.ly/FftCums

- Vásquez, G. (1995). "Ética discursiva, derecho y democracia". En Motta, C. (Comp.). Ética y Conflicto, Lecturas para una transición democrática. pp. 49-81. Bogotá: Tercer Mundo.

- Vásquez, R. (2007). “Corrupción política y responsabilidad de los servidores públicos". DOXA Cuadernos de Filosofía del Derecho, 30, pp.: 205-216. Disponible en https://cutt.ly/VcJV9fg

- Vergara, L. (2011). "Habermas y la Teoría de la Acción Comunicativa". Razón y Palabra, (75), pp.: 1-20. Disponible en https://cutt.ly/MftCiSQ

- Zoffoli, E. (2010). "Jürgen Habermas: Entre hechos y normas". Isonomía, (32), pp.: 69-90. Disponible en https://cutt.ly/ YfyS1Hs 